\title{
Preparation and Characterization of Ordered Nanostructured Cobalt Films via Lyotropic Liquid Crystal Templated Electrodeposition Method
}

\author{
Hassan M. Al-Bishri, Ibrahim S. El-Hallag, ${ }^{\dagger, *}$ and ElSayed H. El-Mossalamy \\ Chemistry Department, Faculty of Science, King Abdul-Aziz University, Jeddah, Saudi Arabia, \\ P.O. Box 80203, Jeddah 21589 \\ ${ }^{\dagger}$ Chemistry Department, Faculty of Science, Tanta University, Tanta 31527,Egypt. "E-mail: ielhallag@yahoo.co.uk \\ Received March 30, 2010, Accepted October 19, 2010
}

\begin{abstract}
A simple, inexpensive and less time consuming electrochemical methods were carried out to prepare ordered mesoporous cobalt films. Ordered mesoporous cobalt films were successfully synthesized by templated electrodepostion of hexagonal $\mathrm{H}_{1}$-e $\mathrm{Co}$ ion. The electrodeposited mesopores films were characterized by scanning electron microscopy (SEM), transmission electron microscopy (TEM), low angle X-ray diffraction (XRD) and voltammetric methods. The applicability of thin films as high - performance super capacitors electrode materials is demonstrated electrochemically using cyclic voltammetry $(\mathrm{CV})$ technique.
\end{abstract}

Key Words: Mesoporous cobalt films, Electrodeposition, Pluronic lyotropic liquid crystal, Hexagonal phase

\section{Introduction}

The preparation of ordered nanostructured mesoporous materials have been extensively investigated in the last decades due to their unique properties that cannot be obtained from the conventional macroscopic materials. ${ }^{1}$ These mesoporous materials exhibit very interesting electrical, optical, magnetic and chemical properties, which could not be achieved by their bulk counterparts. ${ }^{2}$ Mesoporous materials may also be used in various technological applications viz refrigeration systems, medical imaging, drug targeting and other biological applications and catalysis. $^{3}$

In recent years, interest in electrochemical capacitors (ECs) has risen due to their higher power density and longer cycle life compared to secondary batteries and higher energy density that conventional electrical double-layer capacitors. ${ }^{4-6}$ They play increasingly important roles in power source applications such as auxiliary power sources for mobile electronic devices, etc. ${ }^{7,8}$ Electrochemical capacitors utilize both electrical double layer and interfacial redox processes to store energy at an electrode/ electrolyte interface. Accordingly, structural properties are strongly related to the electrochemical properties of Co films for supercapacitors. It is well known that the pore size at the range of $2-50 \mathrm{~nm}$ (mesopores) is considered to be highly desirable for the electrochemical supercapacitors, and the ordered mesoporous make ionic motion easier compared with conventional mesoporous material in which the pores are randomly connected. ${ }^{9}$

It was demonstrated that the lyotropic liquid crystalline phases of polyoxyethelene surfactants can be utilized as versatile nano scale molds for the formation of highly ordered mesoporous materials such as inorganic oxides and metals. ${ }^{10}$ The hexagonal phase composition of the Pluronic P84/Co/p-xylene system was determined according to the phase diagram established in literature. ${ }^{11-13}$ The construction of nanostructured materials using surfactant molecules as templates was found to be a very productive area of research. ${ }^{13,14}$ The idea of using the lyotropic

liquid crystalline phases of nonionic surfactants to template the deposition of inorganic materials was first introduced by Attard who demonstrated the formation of nanostructured silica by hydrolysis of tetramethyl orthosilicate and platinum by chemical reduction of hexachloroplatinic acid. ${ }^{15-17}$ In this case a high concentration of the non surfactant, typically $40-60 \mathrm{wt} \%$ is employed and the nanostructure is a direct cast of the structure of the lyotropic phase used to template the deposition.

In the hexagonal phase the surfactant molecules assemble into long cylindrical micelles and these micelles then pack into a hexagonal arrays (Figure 1) in which the separation between the micelles is comparable to their diameter (around $2 \mathrm{~nm}$ ). ${ }^{18}$ When these phases are used to template the electrochemical deposition of metal films, the metal salt and electrolyte are dissolved into the aqueous component of the mixture and metal deposition occurs from the electrode surface, around the surfactant micelles. When deposition is complete, the surfactant is removed by washing to leave a metal film punctured by a regular hexagonal arrays of uniform pores. The thickness of the film is directly controlled by the total charge passed. Also, the generation of ordered arrays of nanoscale holes in various metallic thin films by using a mask made from a 35-nm-period, 18-nmsphere-diameter spherical morphology block copolymer film and a $\mathrm{CF}_{4}$ plasma etching process that sputter-etches the metal films were demonstrated. ${ }^{19}$ It was concluded that in this work and in the work established in literature, the advantages of the liquid crystal template method are the unchanged topology of

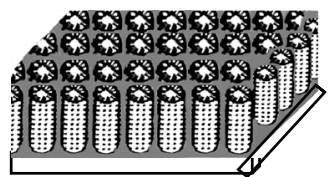

Electrode surface $(\mathrm{Au})$

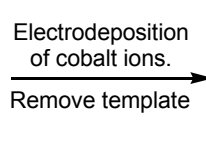

Figure 1. Schematic mechanism of the growth process of the mesoporous $\mathrm{H}_{1}$-e Co films electrodeposited in the presence of hexagonal liquid crystal template. 
the phase throughout the progress of the reaction and the calcinations process and the ability to fabricate thin films with high surface area via electrodepostion. ${ }^{20}$

The present work describes the fabrication of nanostructured mesoporous cobalt films $\left(\mathrm{H}_{1}-\mathrm{eCo}\right)$ by the electrodeposition of cobalt from the aqueous domain of the hexagonal liquid crystal phase of $\mathrm{p}_{84}$. The structure of these $\mathrm{H}_{1}$-eCo films has been investigated using scanning electron microscopy (SEM), transmission electron microscopy (TEM) and X-ray diffraction. Electrochemical capacitance performance of the films was investigated by the means of cyclic voltammetry $(\mathrm{CV})$.

\section{Experimental}

Materials and methods. Plouronic 84 is a trade name for poly (ethylene oxide)-block-poly-(propylene oxide)-block-poly (ethylene oxide) copolymer was obtained from Prof. Bartlett. ${ }^{21}$ All solutions and liquid crystalline template (LCT) were prepared using reagent grade $(18 \mathrm{M} \Omega \mathrm{cm})$. All glassware was cleaned by soaking in 5\% Decon 90 (Aldrich solution for few days followed by rinsing with deionized water and dried in an oven at $50{ }^{\circ} \mathrm{C}$. P-xylene $(99 \%)$, cobalt acetate, potassium acetate and boric acid were obtained from Aldrich. All chemicals were analytical grade and used as obtained without further purification. Cobalt acetate $\left(\mathrm{CoAc}_{2} \cdot 4 \mathrm{H}_{2} \mathrm{O} 99.5 \%\right)$ and potassium acetate (KAc 99\%) were obtained from Fluka.

The plating mixtures used in this work were ternary system consisting of $\mathrm{P}_{84}$ non-ionic surfactant $\left[(\mathrm{EO})_{19}(\mathrm{PO})_{43}(\mathrm{EO})_{19}\right]$, aqueous solution of $\mathrm{CoAc}_{2} \cdot 4 \mathrm{H}_{2} \mathrm{O}, \mathrm{H}_{3} \mathrm{BO}_{3}$ and $\mathrm{p}$-xylene. The $\mathrm{H}_{1}$ phase of ternary mixture containing 53.13 wt $\%$ aqueous $\mathrm{Co}$ salts and boric acid, $44.6 \%$ weight of $\mathrm{P}_{84}$ and $2.23 \%$ weight of p-xylene which is stable at room temperature and remains chemically stable for more than a month. Electrodeposition from the liquid crystalline plating mixture onto polished gold electrode was conducted at room temperature, after deposition the electrodes were rinsed with excess amounts of deionized water to remove the surfactant. The gold electrode was cleaned by sonication in propanol (BDH) for $1 \mathrm{~h}$ followed by rinsing with deionized water. $^{22}$

Instrumentation. The electrochemical experiments were carried out using an EG \& G 283 Potentiostat and a conventional three electrode cell ( $15 \mathrm{~mL}$ volume capacity). In electrochemical measurements, the working electrode was a $1 \mathrm{~mm}$ diameter gold disk electrode, while for the structural characterization of the produced material films, the working electrode was $1 \mathrm{~cm}^{2}$ are flat electrode. Platinum gauze of area $1 \mathrm{~cm}^{2}$ was used as counter electrode and saturated calomel electrode (SCE) as the reference electrode. $1 \mathrm{~mm}$ disk electrode was polished by polishing paper (grade 1200) followed by alumina (Buehler) of two grades: 1.0 and $0.3 \mu \mathrm{m}$ then rinsed with deionized water.

An analytical scanning electron microscope (JOEL 6400) was used to study the morphology and thickness of the electrodeposited cobalt films. Samples for SEM analysis were prepared by electroplating Co from the template mixture on to evaporated gold electrodes (area $1 \mathrm{~cm}^{2}$ ) prepared by evaporation of a $10 \mathrm{~nm}$ thick layer of chromium (to ensure good adhesion) followed by $200 \mathrm{~nm}$ of gold on $1 \mathrm{~mm}$ thick glass microscope slides. These evaporated gold electrodes were cleaned in an ultrasonic bath of 2-propanol for 10 min immediately before use. XRD with $\mathrm{Cu} \mathrm{K} \alpha$ radiation was used to prove primary evidence for the formation of a nanostructured films. The regularity of the nanostructure was investigated using JEOL 2000FX transmission electron microscope operating at an accelerating voltage of $200 \mathrm{KV}$. The nanostructured Co samples were strongly adhered on the gold electrode surface and samples for TEM analysis were prepared by scraping small samples of cobalt off of the evaporated gold electrode surface and on to the TEM grid using a scalpel.

\section{Results and Discussion}

SEM and TEM characterization of cobalt films. It was established that the nanoarchitecture of the final electrochemically deposited film is directly determined by the structure of the lyotropic phase used of deposition. ${ }^{11-13,23}$ The cobalt films which were electrochemically deposited at $-0.90 \mathrm{~V} v s$. SCE from the hexagonal phase template mixture have a silvery metallic luster and adhered well to the evaporated gold electrodes used for the deposition. Figure 2A shows scanning electron micrograph of an $\mathrm{H}_{1}$-eCo film which indicates the cobalt film formed is featureless. The cross-sectional view (Figure $2 \mathrm{~B}$ ) shows that the film is compact, smooth and uniform. The film is about $226 \mathrm{~nm}$ thick. Cobalt films electrodeposited from aqueous acetate solution (Figure 2C) have larger feature size, typically $>1.5 \mu \mathrm{m}^{23,24}$

There is no evidence for nanostructure visible on the scale of the SEM. To examine the nanostructure of the electrochemically deposited cobalt films, it is necessary to use TEM. Figure 3 shows TEM images of the deposited cobalt films at different potentials $(-0.80 \mathrm{~V},-0.85 \mathrm{~V},-0.90 \mathrm{~V}$ and $-0.95 \mathrm{~V})$. It is apparent

(A)

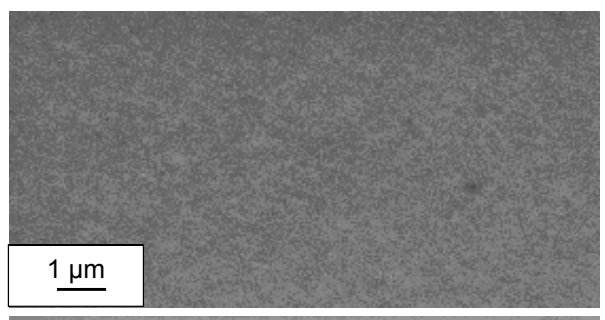

(B)

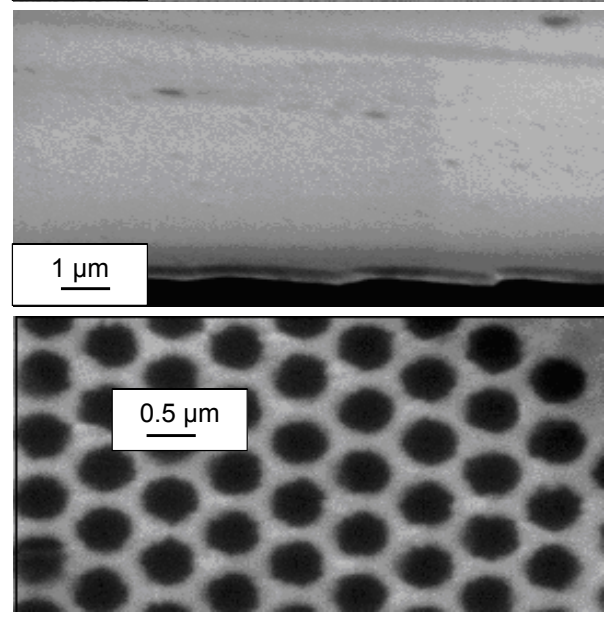

Figure 2. SEM photographs of the $\mathrm{H}_{1}-\mathrm{eCo}$ films deposited at $-0.90 \mathrm{~V}$ vs SCE (A) surface image, (B) cross-section image and (C) film deposited from aqueous solution. 
(A)

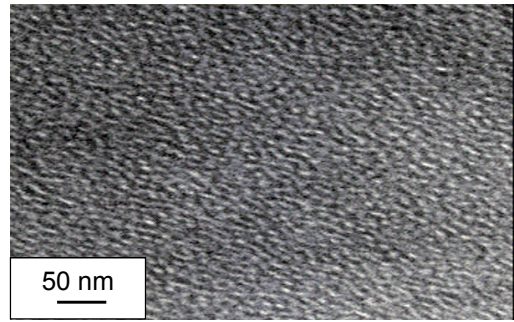

(B)

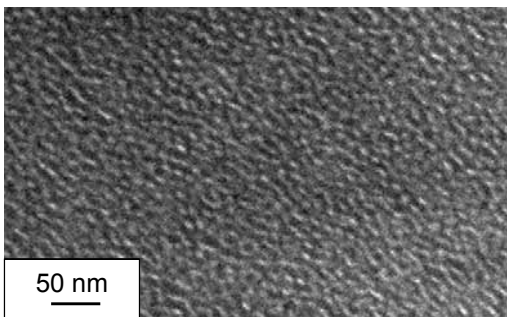

(C)

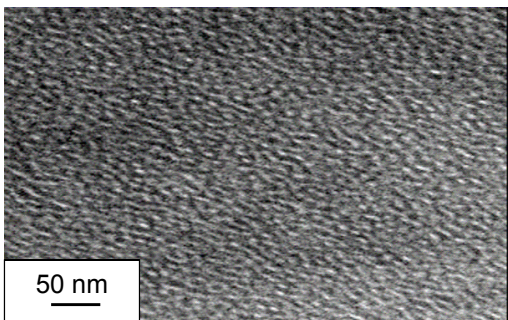

(D)

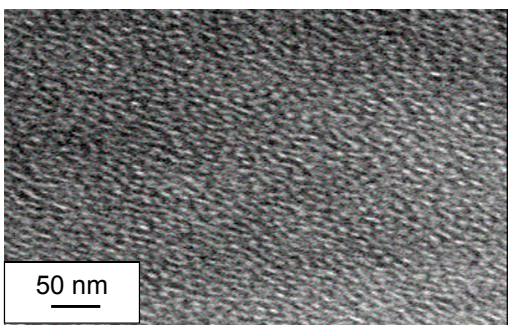

Figure 3. TEM image of the $\mathrm{H}_{1}$-eCo films electrodeposited at various deposition potentials: (A) $-0.80 \mathrm{~V}$; (B) $-0.85 \mathrm{~V}$; (C) $-0.90 \mathrm{~V}$; (D) $-0.95 \mathrm{~V}$.

that all the TEM images have uniform and well ordered hexagonal arrays of mesoporous structure. The bright regions correspond to the pores left after removal of the surfactant template from the electrochemically deposited films. The dark regions correspond to the electrodeposited cobalt metal. From the images it is clear that the pores are hexagonally arranged over length scales. Inspection of these pores show that they are continuous and approximately straight over their whole length, although some defects are evident. These defects and the slight deviation from linearity are expected if the cobalt is a cast of the lyotropic crystalline phase when it is a soft solid. ${ }^{25,26}$ The films are estimated to have a pore center - to center distance of about $9.12 \mathrm{~nm}$, with a uniform pore diameter of about $7.65 \mathrm{~nm}$ and cobalt wall thickness of about $4.5 \mathrm{~nm}$. According to the TEM results, it is noted that the mesostructures of the $\mathrm{H}_{1}$-eCo almost remain unchanged under different deposition potentials, which is consistent with the results reported in literature. ${ }^{27,28}$ On the basis of the pore size and wall thickness, a high specific surface area inside these pores would be expected. This is supported by X-ray investigation.

X-ray characterization of $\mathrm{H}_{1}$-eCo films. Low-angle XRD

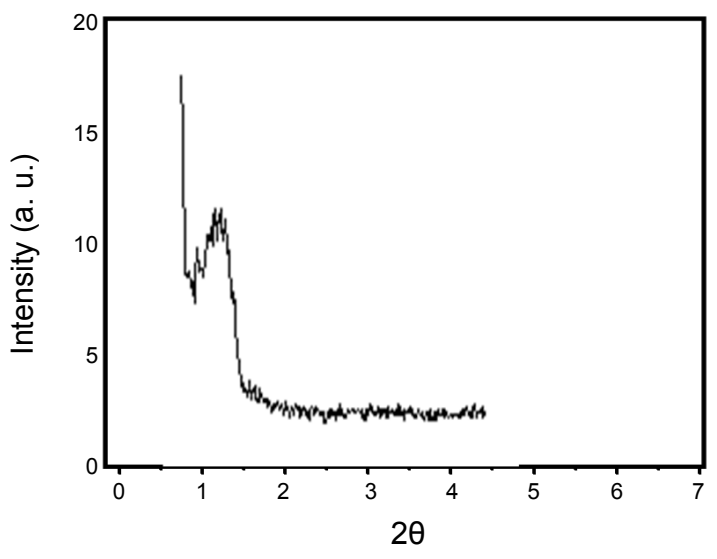

Figure 4. Low -angle XRD for the $\mathrm{H}_{1}$-eCo film electrodeposited on gold substrate from the hexagonal liquid crystal template.

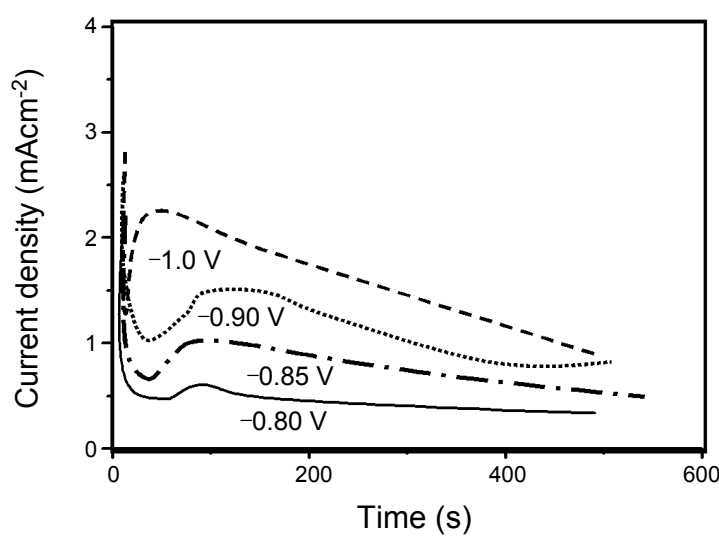

Figure 5. Current time transients for the electrodeposition of $\mathrm{H}_{1}-\mathrm{Co}$ nanostructure cobalt films on $1 \mathrm{~mm}$ diameter gold disk electrode at different deposition potentials from the hexagonal lyotropic liquid crystalline phase mixture.

spectra of the electrodeposited Co film from the electrolyte template of the hexagonal $\left(\mathrm{H}_{1}\right)$ phase shows only one peak at small angle, which indicates the existence of a size controlled nanoporosity. Figure 4 shows an example of the low angle XRD spectra. The low angle $\mathrm{XRD}$ of the hexagonal $\mathrm{H}_{1}$-eCo film displays diffraction peak at about $2 \theta=1.11^{\circ}$ corresponding to the (100) diffraction phase. It was found that the $\mathrm{d}_{100}$-spacing is equals to $79.58 \mathrm{~A}^{\mathrm{o}}$ as expected for the $2 \mathrm{D}$ hexagonal mesophase. The pore -to- pore distance $(\mathrm{R})$ for this hexagonal arrays given by:

$$
\mathrm{R}=\mathrm{d}_{110} / \cos 30
$$

is $9.19 \mathrm{~nm}$.

The basic vector length (lattice parameter), $a_{h}$ of the hexagonal phases can be obtained by using the following relation: ${ }^{29}$

$$
a_{h}=(2 d / \sqrt{ } 3)
$$

As calculated from Eq.(2), the distance between two centers of adjacent cylinders, $a_{h}$ for the pure hexagonal phase is $9.19 \mathrm{~nm}$ 
(A)

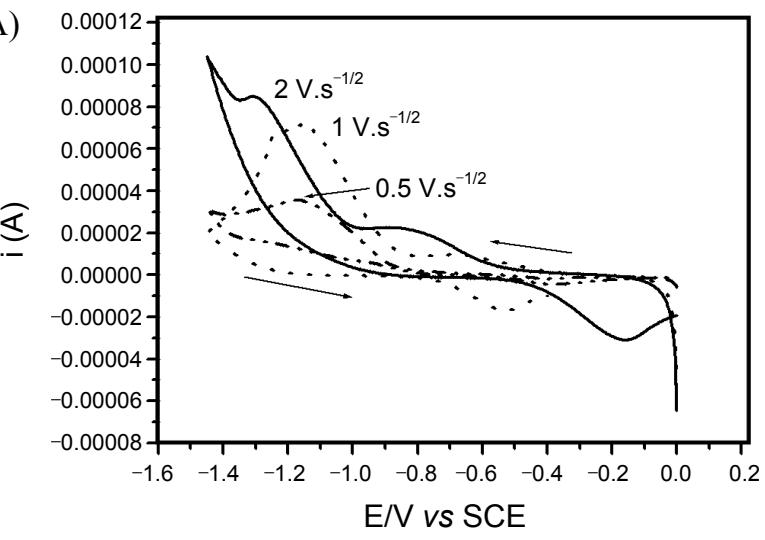

(B)

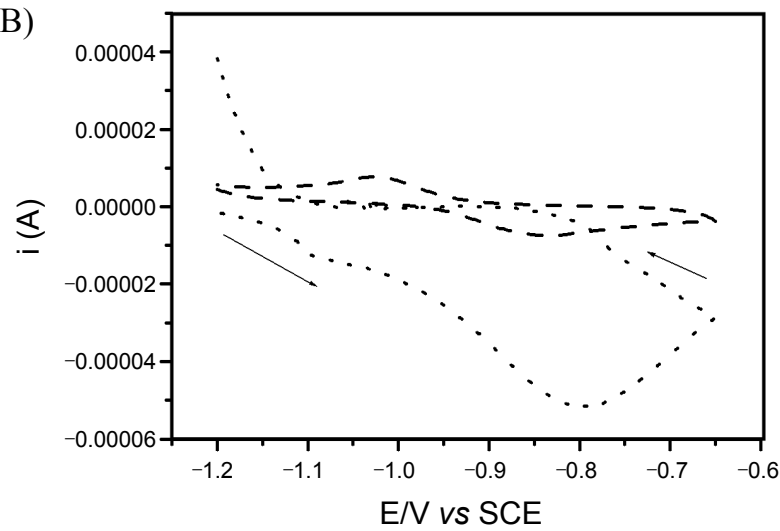

Figure 6. Cyclic voltammograms of $0.2 \mathrm{M} \mathrm{CoAc}$ in pluronic lyotropic liquid crystal at different scan rates (A) and of deposited cobalt films from aqueous media (----) \& from pluronic lyotropic liquid crystal (..........) (B) in $0.1 \mathrm{M} \mathrm{NaOH}$ at sweep rate of $10 \mathrm{mV} . \mathrm{s}^{-1}$.

agrees well with the value calculated from Eq.(1). The appearance of low angle peal indicates that mesoscopic order in the liquid crystalline template is preserves in the nanoporous Co films. The broading of the single films diffraction peak may be due to a partial destruction of long-range order during electrodeposition.

Current time transients for deposition of $\mathrm{H}_{1}$-eCo. Figure 5 shows typical current time transients for electrochemical deposition of cobalt from the hexagonal lyotropic $\left(\mathrm{H}_{1}\right)$ phase at deposition potentials $-0.80 \mathrm{~V},-0.85 \mathrm{~V},-0.9 \mathrm{~V}$ and $-1.0 \mathrm{~V} v s$ SCE. The deposition transients, show an initial current decay with time, which can be attributed to double layer charging followed by an increase in the current to a broad maximum and then decay. Films grown at potentials of $-0.85 \mathrm{~V}$ to $-0.90 \mathrm{~V}$ were silver and highly reflective. In all subsequent experiments the cobalt films were deposited by potential step to $-0.90 \mathrm{~V}$.

Cyclic voltammetry of electrodeposited $\mathrm{H}_{1}$-eCo films. Cyclic voltammetry measurements have been used to evaluate the electrochemical properties of the hexagonal $\mathrm{H}_{1}-\mathrm{eCo}$ film. The surface faradaic reaction of the deposited Co proceeded according to the following anodic reaction:

$$
\mathrm{Co} \leftrightarrow \mathrm{Co}^{2+}+2 \mathrm{e}
$$

Figure 6A shows the characteristic CV curves of the cobalt ion in pluronic lyotropic liquid crystal template at different scan
Table 1. Electrochemical characterization of CoAc in pluronic lyotropic liquid crystal template and of $\mathrm{H}_{1}$-eCo films deposited in $1 \mathrm{M}$ $\mathrm{NaOH}$

\begin{tabular}{lcrc}
\hline \multicolumn{1}{c}{ Co/electrolyte } & $\mathrm{v} / \mathrm{mV} \cdot \mathrm{s}^{-1}$ & $\mathrm{ip} / \mathrm{uA}$ & Peak area/uA $\cdot \mathrm{V}$ \\
\hline $\mathrm{Co}^{2+}$ in Lyotropic & 20 & 5.86 & 1.687 \\
liquid crystalline & 50 & 8.40 & 3.810 \\
template & 100 & 14.10 & 5.260 \\
& 200 & 20.50 & 11.754 \\
$\mathrm{H}_{1}$-eCo film & 10 & $8.20^{a}$ & 2.100 \\
& & $51.8^{b}$ & 26.400 \\
\hline
\end{tabular}

$\overline{{ }^{a} \mathrm{CV} \text { data of deposited film from aqueous media in } 1 \mathrm{M} \mathrm{NaOH} .{ }^{b} \mathrm{CV} \text { data }}$ of deposited film from lyotropic crystalline media in $1 \mathrm{M} \mathrm{NaOH}$.

rates. It is clear to see that the pair of redox peaks is visible in each CV curve, indicating that the capacitance is mainly based on the redox mechanism. ${ }^{28}$ The cathodic peak is due to the reduction of $\mathrm{Co}^{2+}$ ion to Co metal and the anodic peak is for the reverse process. Furthermore, the shape of the $\mathrm{CV}$ curves reveals that the capacitance characteristic of Co is distinct from that of the electric double layer capacitance, which would produce a $\mathrm{CV}$ curve close to an ideal rectangular shape. Since electrolyte template and electrode resistance can distort current response at the switching potential and this distortion is dependent upon the scan rate, ${ }^{30}$ the shape of the $\mathrm{CV}$ has changed with variation of the scan rate increased. These results indicate that the capacitance is mainly based on redox mechanism. Figure $6 \mathrm{~B}$ indicates the cyclic voltammograms of the $\mathrm{H}_{1}$-eCo films electrodeposited from aqueous media and from liquid crystalline template. It was observed that the cyclic voltammogram in case of liquid crystalline template has a surface area higher than that in case of the aqueous media confirming the increase of the specific capacitance of the $\mathrm{H}_{1}$-eCo film in lyotropic liquid crystal template. Table 1 summarized the obtained data of the electrochemical characterization of the investigated system. As shown, the data cited in Table 1 revealed increasing the electrode surface area of the hexagonal crystalline template more than that in the case of aqueous medium leading to increasing the capacitance of the $\mathrm{H}_{1}$-eCo film.

\section{Conclusion}

The mesoporous $\mathrm{H}_{1}$-eCo films is successfully electrodeposited on the gold substrate from the hexagonal lyotropic liquid crystalline phase of an inexpensive nonionic surfactant pluronic 84 (P84). All the results indicate that the experimental conditions have significant effect on the structure and electrochemical capacitance of the prepared $\mathrm{H}_{1}$-eCo films. The results obtained from TEM and low angle XRD indicate that the presence of nanostructure and support a direct templating mechanism for the electrodeposition of mesoporous Co films, which implies that the Co films have higher aspect ratio and excellent mesoporous films exhibit high specific capacitance, which may be attributed to a larger effective surface area within mesoporous films. Electrodeposition from lyotropic liquid crystalline phases provides a new templating approach has potential application to fabricate supercapacitor materials. 
Acknowledgments. The author is grateful to Prof. P. N. Bartlett, School of Chemistry, University of Southampton, UK, for providing materials for this work.

\section{References}

1. Khiew, P. S.; Radiman, S.; Huang, N. M.; Ahamd, Md S. J. Crystal Growth 2004, 268, 227

2. Colvin, V. L.; Schlamp, M. C.; Alivisatos, A. P. Nature 1994, 370, 354

3. Kesavan, V.; Sivanand, S. P.; Chandrasekaran, S.; Kaltypin, Y.; Gedanken, A. Angew. Chem. Int. 1999, 38, 3521.

4. Conway, B. E. J. Electrochem. Soc. 1991, 138, 1539.

5. Trasatti, S.; Kurzweil, P. Platinum Met. Rev. 1994, 38, 46.

6. Sarangapani, S.; Tilak, B. V.; Chen, C. P. J. Electrochem. Soc. 1996, 143, 3791.

7. Andrew, A. J. Power Source 2000, 91, 37.

8. Zhao, D. D.; Xu, M. W.; Zhou, W. J.; Zhang, J.; Li, H. L. Electrochim. Acta 2008, 53, 2699.

9. Yoon, S.; Lee, J.; Hyeon, T.; Oh, S. M. J. Electrochem. Soc. 2000, $147,2507$.

10. Nandhakumar, I.; Elliott, J. M.; Attard, G. S. Chem. Mater. 2001, 13,3840

11. Raman, K. N.; Anderson, M. T.; Brinker, C. J. Chem. Mater. 1996, $8,1682$.

12. Paavola, A.; Ylirusi, J.; Rosenberg, P. J. Control. Rel. 1998, 52, 169

13. Liu, J.; Shin, Y.; Nie, Z.; Chang, J. H.; Wang, L. Q.; Fryxell, G.
E.; Samuels, W. D.; Exarhos, G. J. J. Phys. Chem. 2000, A 104, 8328.

14. Raimondi, M. E.; Seddon, J. M. Liq. Cryst. 1999, 26, 305.

15. Attard, G. S.; Goeltller, C. G.; Corker, J. M.; Henke, S.; Templer, R. H. Angew. Chem. Int. Ed. Engel. 1997, 36, 1315.

16. Bartlett, P. N.; Gollas, B.; Gurin, S.; Marrwan, J. Phys. Chem. Chem. Phy. 2002, 4, 3835.

17. Attard, G. S.; Glyde, J. C.; Goeltner, C. G. Nature 1995, 378, 366.

18. Mitchell, D. J.; Tiddy, G. J. T.; Waring, J.; McDonald, T. J. Chem. Soc. Faraday Trans. 1983, 79, 975

19. Jung, Y. S.; Ross, C. A. Small 2009, 5, 1654.

20. Dong, B.; Xue, T.; Xu, C.-L.; Li, H.-L. Microporous Mesoporous Mater. 2008, 112, 627.

21. Bartlett, P. N. University of Southampton, Private communication.

22. Bartlett, P. N.; Birikin, P. R.; Ghanem, M. A.; Groot P. de.; Sawicki, M. J. Electrochem. Soc. 2001, 148, C119.

23. El-Hallag, I. S. Bull. Mater. Sci. 2009, 32, 555.

24. Fan, C. L.; Piron, D. L.; Miao, H. J.; Rojas, M. J. Appl. Electrochem. 1993, 23, 985.

25. Wang, L.; Shi, J.; Yu, J.; Yan, D. Nanostructure Mater. 1998, 10 , 1289.

26. Karakasside, M. A.; Bourlions, A.; Petridis, D.; Coche-Guerente, L.; Labbe, P. J. Mater. Chem. 2009, 10, 403.

27. Tan, Y.; Srinivasan, S.; Choi, K. S. J. Am. Chem. Soc. 2005, 127 , 3596.

28. Zhou, W. J.; Zhao, D.-D.; Xu, M-W.; Xu, C.-L.; Li, H-L. Electrochim. Acta 2008, 53, 7210.

29. Lee, M. H.; Oh, S. G.; Suh, K. D.; Kim, D. G.; Sohn, D. Colloid Surf. 2002, A210, 49.

30. Jiang, J. H.; Kucernak, A. Electrochim. Acta 2002, 47, 2381. 